\section{Синтез схемы управления гидроманипулятором}

\author{
Нестеров Л.А. '
}

Петрозаводский государственньий университет

В данной работе рассматривается один из методов синтеза схемы управления гидроманипулятором по стандартным траекториям перемешения его стрелы с помощью таблиц вычисления, показывающих взаимодействие командных и исполнительных элементов в течение цикла.

Ключевые слова: гидроманипулятор, траектория, датчики, таблицы, схемы, элемент, формула, зоны, включения, выключения.

К механизмам, позволяющим полностью исключить ручной труд на разгрузке и разборке пачек хлыстов, относится гидроманипулятор. В настояшее время на лесных складах применяют различные конструкции таких манипуляторов. Управление ими производится при помощи гидравлических систем. главными элементами которых являются гидроцилиндры, управляемые золотниками. Рабочий процесс управления гидроцилиндрами состоит из периодически повторяющихся циклов ручного включения и выключения золотников (до 500 срабатываний в час), что приводит к быстрому утомлению оператора. В связи с этим возникает задача внедрения автоматизированного управления манипулятором.

Трудность разработки схемы управления гидроманипулятором связана с неопределенностью траектории движения его стрелы, что требует ручного управления. Однако после захвата хлыста появляется возможность автоматического управления стрелой, так как она перемещается по стандартной траектории, например, согласно траектории. приведенной на рис. 1

Для автоматического управления гидроманипулятором по стандартным траекториям необходима информация о нахождении стрелы в той или иной точки траектории. Это может быть осуществлено при помощи датчиков положения, установленных в точках перехода стрелы с одного направления перемещения на другое.

В данной работе рассматривается один из методов синтеза схемы управления гидроманипулятором по стандартным траекториям перемешения его стрелы с помощью таблиц включения. показывающих взаимодействие командных и исполнительных элементов в течение цикла.

Из траектории движения (рис.1,a) видно, что при одном и том же положении стрелы в точке $\left(\mathrm{x}_{2}, \mathrm{y}_{1}\right)$ должны быть поданы команда 3 на движение вниз и команда 5 на движение вправо (рис. 1,6$)$.

1 Автор - дочент кафедры технологии и оборудования лесного комплекса

(C) Л.А.Нестеров, 1996
Для устранения этой неопределенности необходимы дополнительные однозначные условия. Этими дополнительными условиями могут быть положения стрелы гидроманипулятора в предыдущих точках траектории ее движения, т.е. схема должна иметь запоминающие элементы, что требует применения методов синтеза многотипных схем управления.

Запишем цикл работы гидроманипулятора по стандартной траектории в виде таблицы включения. Для составления таблицы включения введем обозначения:

$\mathrm{x}_{1}$ - датчик положения стрелы в точке $\left(\mathrm{x}_{1}, \mathrm{y}_{1}\right)$;

$\mathrm{x}_{2}$ - датчик положения стрелы в точке $\left(\mathrm{x}_{2}, \mathrm{y}_{1}\right)$;

$\mathrm{x}_{3}$ - датчик положения стрелы в точке $\left(\mathrm{x}_{2}, \mathrm{y}_{2}\right)$;

П - электромагнит гидрозолотника, управляющий перемещением стрелы вправо;

$\Lambda$ - электромагнит гидрозолотника, управляюший перемещением стрелы влево;

H - электромагнит гидрозолотника, управляющий перемещением стрелы вниз;

В - электромагнит гидрозолотника, управляющий перемещением стрелы вверх

Таблица включения, характеризующая работу гидроманипулятора по указанной траектории, приведена на рғс. 2. В горизонтальные строки таблицы вписаны все элементы управления (Э). Вертикальные столбцы - это такты (Т). Знаком "+" обозначается включение элемента, знаком "-" его выключение

Таблица включения является основой синтеза схем управления. Она позволяет определять возможность реализации цикла и количество промежуточных элементов.

С цельго определения возможности реализации цикла каждому элементу в таблице включения присваивается так называемый вес. Если всем элементам, входящим в таблицу включения, присвоить порядковые номера с нуля до $\mathrm{n}$, то весом элемента является число $2^{\mathrm{n}}$. Так, нулевому элементу присваивается вес $2^{0}=1$; первому элементу $2^{\prime}=2$; в торому $-2^{2}=4$; третьему $-2^{3}=8$ и т.д.

Сумма весов элементов называется весом состояния. Если веса состояний во всех тактах таблицы включения различны, то таблица включения реализуема, т.е. для данного цикла схема может быть выполнена без применения дополнительных цикловых промежуточных элементов. Если же в тактах таблицы включения имеются повторения весов состояний, то такая таблица является нереализуемой и необходимо ввести промежуточные элементы для.того, чтобы устранить это повторение.

Из таблицы на рис. 2 видно, что повторения весов состояний имеются в тактах 1 и 17, а также в тактах 5 и 13. Следовательно, эта таблица не может быть реализована в виде релейной схемы без применения промежуточных элементов. Так как указанные зоны являются совмещенными, то для реализации всех нереализующих тактов достаточно одного промежуточного элемента, который должен менять одно состояние внутри наименьшей зоны нереализуемости, а другое - вне наи- 
большей зоны нереализуемости. Это видно из общей таблицы включения на рис. 3 при помощи одного промежуточного элемента а.

Из структурной теории релейных устройств известны общая формула для определения первоначальной структуры какого-либо элемента $\mathrm{X}$ :

$$
F(x)=f_{c p}+x f_{\text {отп }},
$$

где

$\mathrm{f}_{\mathrm{cp}}$ - логическое произведение элементов в тактах срабатывания:

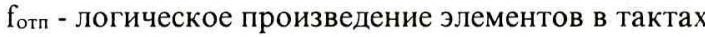
отпускания.

На основе общей таблицы включения схемы управления гидроманипулятором по стандартной траектории определим структурные формулы для исполнительных и промежуточных элементов:

$$
\begin{aligned}
& F(\Pi)=x_{1} \bar{a}+\Pi \overline{\left(x_{2} \overline{x_{1}}\right)}=x_{1} \bar{a}+n\left(\overline{x_{2}}+x_{1}\right), \\
& F(H)=x_{2} \bar{n}+\Pi \overline{\left(\overline{x_{2}} x_{3}\right)}=x_{2} \bar{n}+H\left(x_{2}+\overline{x_{3}}\right), \\
& F(B)=x_{3} \bar{H}+B \overline{\left(\overline{x_{3}} \cdot a \cdot x_{2}\right)}=x_{3} \bar{H}+B\left(x_{3}+\bar{a}+\overline{x_{2}}\right) \text {, } \\
& F(\wedge)=\bar{B}+\wedge\left(\overline{\left.\overline{x_{2}} x_{1}\right)}=\bar{B}+\wedge\left(x_{2}+\overline{x_{1}}\right),\right. \\
& \mathrm{F}(\mathrm{a})={ }_{\mathrm{B}} \overline{\mathrm{X}_{2}}+\mathrm{a} \overline{\left(\bar{\Lambda} \cdot \overline{\mathrm{x}_{1}}\right)}=\mathrm{B} \overline{\mathrm{x}_{2}}+\mathrm{a}\left(\Lambda+\mathrm{x}_{1}\right) .
\end{aligned}
$$

Общая структурная формула для схемы управления может быть представлена в виде

$$
\begin{aligned}
& F=x_{1} \bar{a}+\Pi\left(\overline{x_{2}}+x_{1}\right) \Pi+x_{2} \bar{n}+H\left(x_{2}+\overline{x_{3}}\right) H+x_{3} \bar{H}+ \\
& +B\left(x_{3}+\bar{a}+\overline{x_{2}}\right) B+\bar{B}+\Lambda\left(x_{2}+\overline{x_{1}}\right) \Lambda+B \overline{x_{2}}+a\left(\Lambda+x_{1}\right) A .
\end{aligned}
$$

Структурная схема, соответствующая этой формуле, приведена на рис. 4. Эта схема является последовательно-параллельной (класс П). Однако наи- более экономичными по числу элементов являются схемы с мостовыми соединениями (класс Н). На рис.5 приведена такая схема управления на постоянном токе с исключением ложных цепей при помощи диодов. В соответствии с этой структурной схемой на рис. 6 приведена принципиальная схема управления гидроманипулятором по стандартной траектории в релейном контактном исполнении.

Рассмотренный пример позволяет сформулировать алгоритм синтеза схемы управления гидроманипулятором по стандартной программе.

1. На основании словесного описания цикла работы составляется таблица включения, отражающая зависимость работы командных и исполнительных элементов

2. По таблице включения определяется ее реализуемость, вводятся необходимые промежуточные элементы и составляется общая таблица включения

3. По общей таблице включения определяются первоначальные структурные формулы исполнительных и промежуточных элементов.

4. Упрощение структурных формул осуществляется при помощи алгебры релейных схем.

5. Вычерчивается структурная схема и анализируется ее работа.

6. С учетом выбранных технических средств строится принципиальная схема управления.

После проведения синтеза по приведенному алгоритму имеются еще возможности улучшить схему и уменьшить количество контактов в ней. К ним относятся замена одних контактов равносильными другими, создание мостиковых структур, переход к схемам на постоянном токе и др.

Указанный алгоритм проектирования схем автоматики может быть применен и для других траекторий движения стрелы гидроманипулятора.

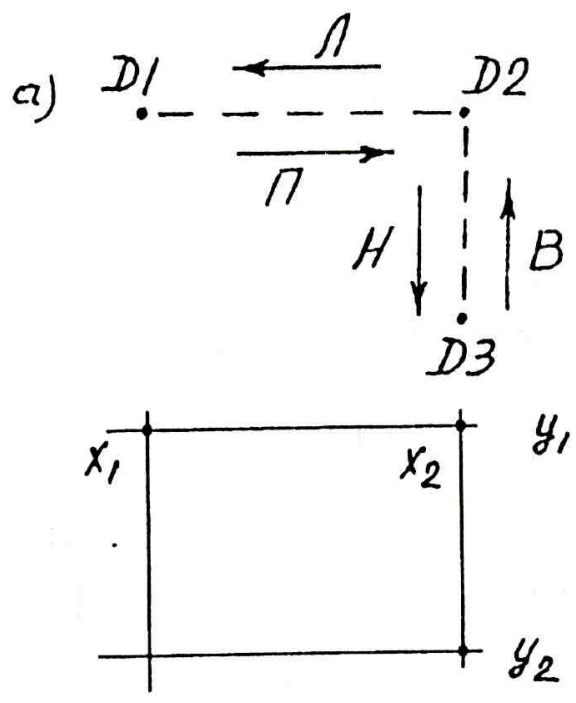

反)

\begin{tabular}{|c|c|c|}
\hline $\begin{array}{c}\mathrm{N} \\
\text { команд }\end{array}$ & Положение & $\begin{array}{c}\text { Направление } \\
\text { движения }\end{array}$ \\
\hline 1 & - & ру \\
2 & $\mathrm{x}_{1} \cdot \mathrm{y}_{1}$ & + \\
3 & $\mathrm{x}_{2} \cdot \mathrm{y}_{1}$ & $\downarrow$ \\
4 & $\mathrm{x}_{2} \cdot \mathrm{y}_{2}$ & $\uparrow$ \\
5 & $\mathrm{x}_{2} \cdot \mathrm{y}_{1}$ & $\rightarrow$ \\
6 & - & $\mathrm{Py}$ \\
\hline
\end{tabular}

Рис. 1. Траектория движения стрелы манипулятора. 


\begin{tabular}{|l|l|l|l|l|l|l|l|l|l|l|l|l|l|l|l|l|l|l|l|l|}
\hline Вес & Э & 0 & 1 & 2 & 3 & 4 & 5 & 6 & 7 & 8 & 9 & 10 & 11 & 12 & 13 & 14 & 15 & 16 & 17 & 18 \\
\hline $\mathrm{l}$ & $\mathrm{x}_{1}$ & $-\mathrm{x}_{1}$ & $+\mathrm{x}_{1}$ & & $-\mathrm{x}_{1}$ & & & & & & & & & & & & & $+\mathrm{x}_{1}$ & & $-\mathrm{x}_{1}$ \\
\hline 2 & $\mathrm{x}_{2}$ & $-\mathrm{x}_{2}$ & & & & $+\mathrm{x}_{2}$ & & & $-\mathrm{x}_{2}$ & & & & & $+\mathrm{x}_{2}$ & & & $-\mathrm{x}_{2}$ & & & \\
\hline 4 & $\mathrm{x}_{3}$ & $-\mathrm{x}_{3}$ & & & & & & & & $+\mathrm{x}_{3}$ & & $-\mathrm{x}_{3}$ & & & & & & & & \\
\hline 8 & $\Pi$ & $-\Pi$ & & $+\Pi$ & & & $-\Pi$ & & & & & & & & & & & & & \\
\hline 16 & $Л$ & $-Л$ & & & & & & & & & & & & & & $+Л$ & & & $-\Omega$ & \\
\hline 32 & $\mathrm{H}$ & $-\mathrm{H}$ & & & & & & $+\mathrm{H}$ & & & $-\mathrm{H}$ & & & & & & & & & \\
\hline 64 & В & $-\mathrm{B}$ & & & & & & & & & & $+\mathrm{B}$ & & & $-\mathrm{B}$ & & & & & \\
\hline $\begin{array}{l}\text { Вес со- } \\
\text { стояния }\end{array}$ & 0 & 1 & 9 & 8 & 10 & 2 & 34 & 32 & 36 & 4 & 68 & 64 & 66 & 2 & 18 & 16 & 17 & 1 & 0 \\
\hline
\end{tabular}

Рис.2. Таблица включения командных и исполнительных элементов

\begin{tabular}{|l|l|l|l|l|l|l|l|l|l|l|l|l|l|l|l|l|l|l|l|l|l|l|}
\hline Вес & Э & 0 & 1 & 2 & 3 & 4 & 5 & 6 & 7 & 8 & 9 & 10 & 11 & 12 & 13 & 14 & 15 & 16 & 17 & 18 & 19 & 20 \\
\hline 1 & $\mathrm{x}_{1}$ & $-\mathrm{x}_{1}$ & $+\mathrm{x}_{1}$ & & $-\mathrm{x}_{1}$ & & & & & & & & & & & & & $+\mathrm{x}_{1}$ & & & - & \\
\hline 2 & $\mathrm{x}_{2}$ & $-\mathrm{x}_{2}$ & & & & $+\mathrm{x}_{2}$ & & & $-\mathrm{x}_{2}$ & & & & & & $+\mathrm{x}_{2}$ & & & $-\mathrm{x}_{2}$ & & & & \\
\hline 4 & $\mathrm{x}_{3}$ & $-\mathrm{x}_{3}$ & & & & & & & & $+\mathrm{x}_{3}$ & & & $-\mathrm{x}_{3}$ & & & & & & & & & \\
\hline 8 & $\mathrm{a}$ & $-\mathrm{a}$ & & & & & & & & & & & & $+\mathrm{a}$ & & & & & & & & $-\mathrm{a}$ \\
\hline 16 & П & $-\Pi$ & & + П & & & $-\Pi$ & & & & & & & & & & & & & & & \\
\hline 32 & Л & $-\mathrm{Л}$ & & & & & & & & & & & & & & & & & & & & \\
\hline 64 & $\mathrm{H}$ & $-\mathrm{H}$ & & & & & & $+\mathrm{H}$ & & & $-\mathrm{H}$ & & & & & & & & & & & \\
\hline $\begin{array}{l}128 \\
\text { В }\end{array}-\mathrm{B}$ & & & & & & & & & & $+\mathrm{B}$ & & & & $-\mathrm{B}$ & & & & & & \\
\hline $\begin{array}{l}\text { Вес } \\
\text { состоя } \\
\text { ния }\end{array}$
\end{tabular}

Рис.3. Общая таблица включения схемы управления

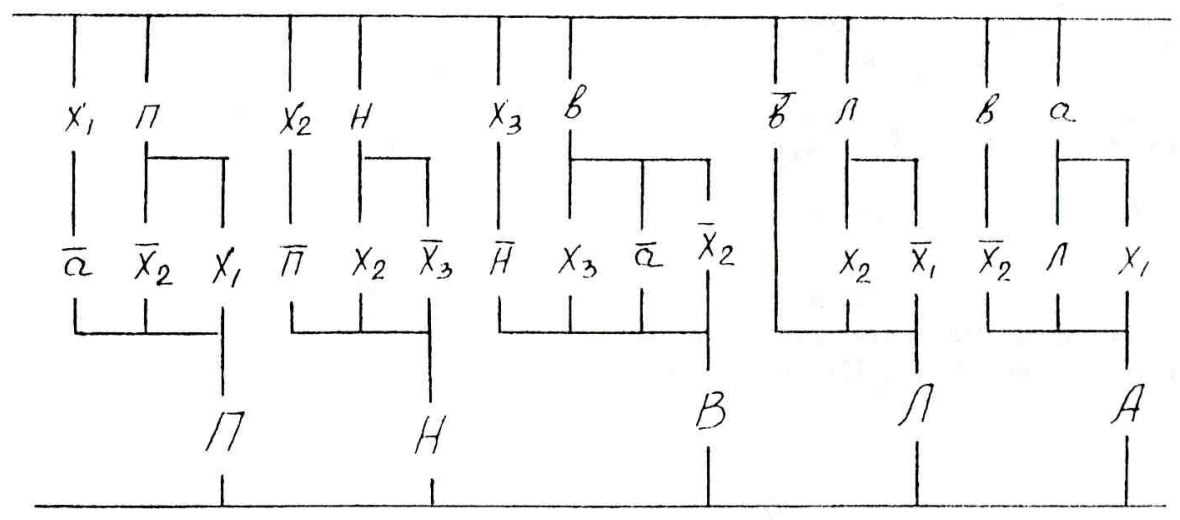

Рис 4. Последовательно-параллельная структурная схема управления

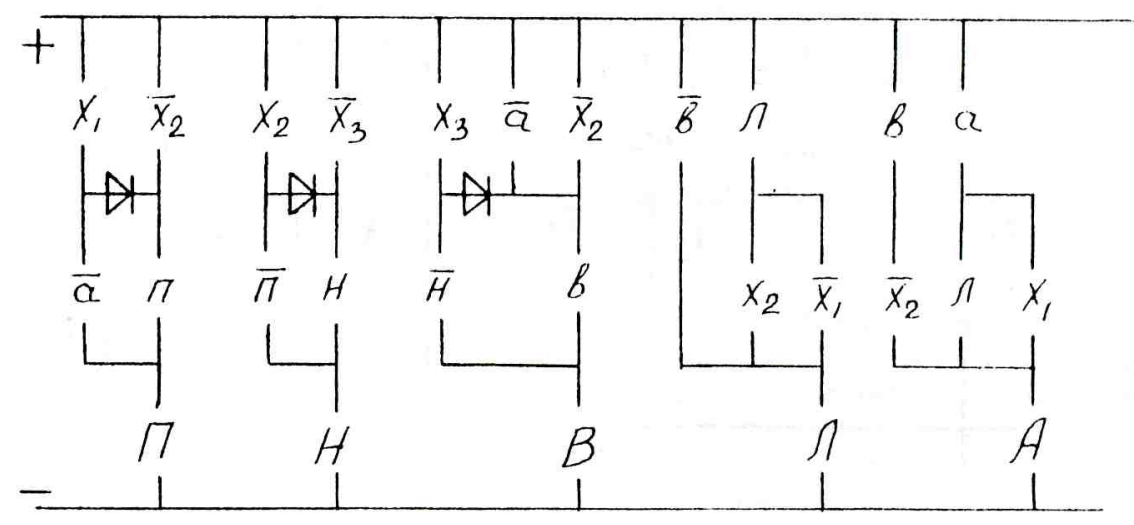

Рис.5. Мостиковая структурная схема управления 


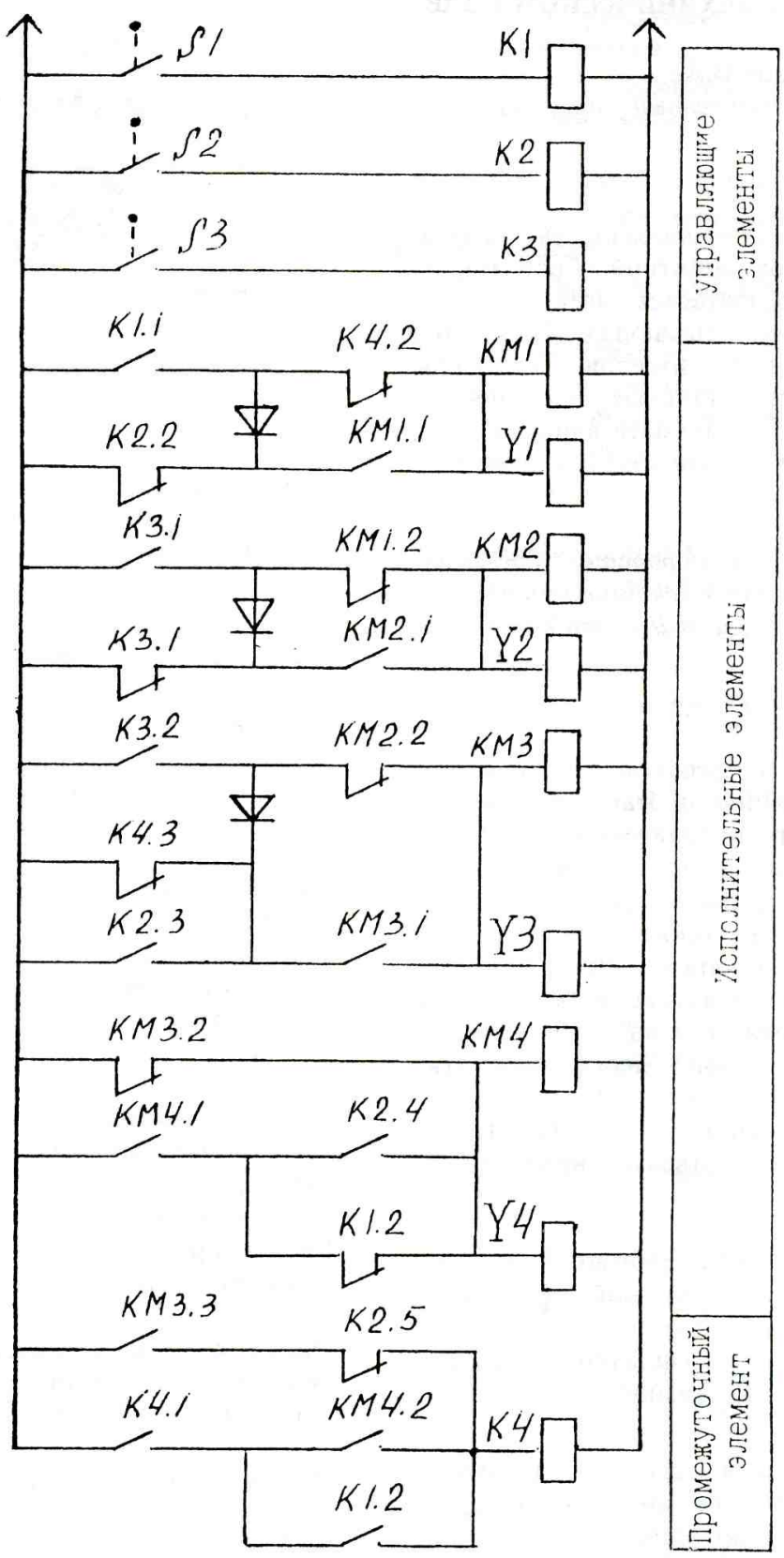

Рис. 6. Принципиальная схема управления гидроманипулятором

\section{ЛИТЕРАТУРА}

1. Вищке Г.А. Автоматизация производственных процессов лесопромышленных предприятий. М.: Лесная промышленность, 1972.413 с.
2. Минасер Э.И.,Сущев М.И. Разработка релейноконтактных схем управления производственных механизмов. М.: Энергия, 1972. 134c. 